\title{
An Unusual Case of Thymoma Invading SVC Excised Completely Enbloc with SVC Resection and Reconstruction
}

\author{
Muhammad Asghar Nawaz, Isaac Kadira and Rajesh Shaha \\ Department of Cardiothoracic and Transplant Surgery, Wythenshawe Hospital NHS, UK
}

\begin{abstract}
We report a challenging rare case of stage III large thymoma with superior vena cava (SVC) invasion but without SVC syndrome or myasthenic symptoms. The tumor responded poorly to neo-adjuvant VAC chemotherapy so was referred for surgical resection. We successfully performed the complex Enblock resection of the tumor with SVC, lung, phrenic and pericardium without requiring cardiopulmonary bypass followed by reconstruction of the SVC and innominate vein with the Gore-Tex graft. The histopathology revealed it to be completely resected type B3 thymoma T3NO. A brief account of literature for advanced thymoma has been provided with this case report.
\end{abstract}

Keywords

Thymoma, SVC syndrome, SVC reconstruction, Gore-Tex graft

\section{Introduction}

Thymomas are rare [1] but account for $20 \%$ of all anterior mediastinal tumors and may often infiltrate the adjacent structures though intraluminal extension is relatively sporadic. Neoadjuvant chemotherapy followed by surgery and radiotherapy increases the R0 resection and 5-year survival rate. We performed a complex operation of enbloc resection followed by distinctive SVC reconstruction with good outcome.

\section{Case Report}

A 69-year-old lady, ex-smoker was found to have a mediastinal mass on an X-ray done for persistent cough. She had no stridor, myasthenia or SVC syndrome clinically but CT revealed $93 \times 58 \mathrm{~mm}$ thymic tumor with definite proximal SVC invasion (Figure 1). Following MDT discussion she was given 4 cycles of Etoposide/Cisplatin chemotherapy but the interval CT scan revealed no real reduction in size so the patient had further 2 cycles of VAC chemotherapy with poor response. Ultimately, she was referred for surgical resection.

We performed acomplex operation via median sternotomy with cervical extension for better exposure and enbloc resection of the thymic tumor with SVC, wedge resection of right upper lobe, part of pericardium and sacrificed the right phrenic nerve to achieve the complete resection. The cardiopulmonary bypass was ready on standby and no systemic heparinization used. Following tumor resection the SVC and left-sided innominate veins were then reconstructed by constituting the T-graft using the $15 \mathrm{~mm}$ non-ringed Gore-Tex graft (Figure 2) without requiring the cardiopulmonary bypass. The distal end-to-side anastomosis was constructed first after partial occlusion of the right internal jugular vein with the side biting clamp followed by proximal anastomosis end-to-side with the right atrial appendage after exclusion of pectinate fibers. Finally, the left sided innominate was anastomosed end-to-end with the horizontal part of the T graft. All vascular anastomosis were constructed using the $5 / 0$ prolene continuous sutures. The operation was uneventful and she made a very good recovery and discharged home on Apixaban.

The post-op CT (Figure 3) showed nicely seated and patent $t$-tube graft and patient was then referred back to oncology for the consideration of radiotherapy as the final histopathology revealed it to be Masaoka stage III, type B3 epitheliod thymoma T3NO but R1 resection due to microscopic tumors close to the resection margin.

\section{Conclusion}

Surgical resection of advanced stage III thymomas in-

*Corresponding author: Muhammad Asghar Nawaz, Department of Cardiothoracic and Transplant Surgery, Wythenshawe Hospital NHS, South Moor Road, Manchester, M23 9LT, UK, Tel: +44-161-2912565, Fax: +44-161-2912685

Accepted: July 11, 2020

Published online: July 13, 2020

Citation: Nawaz MA, Kadira I, Shaha R (2020) An Unusual Case of Thymoma Invading SVC Excised Completely Enbloc with SVC Resection and Reconstruction. J Cardiothorac Surg Ther 2020:4(1):58-60 


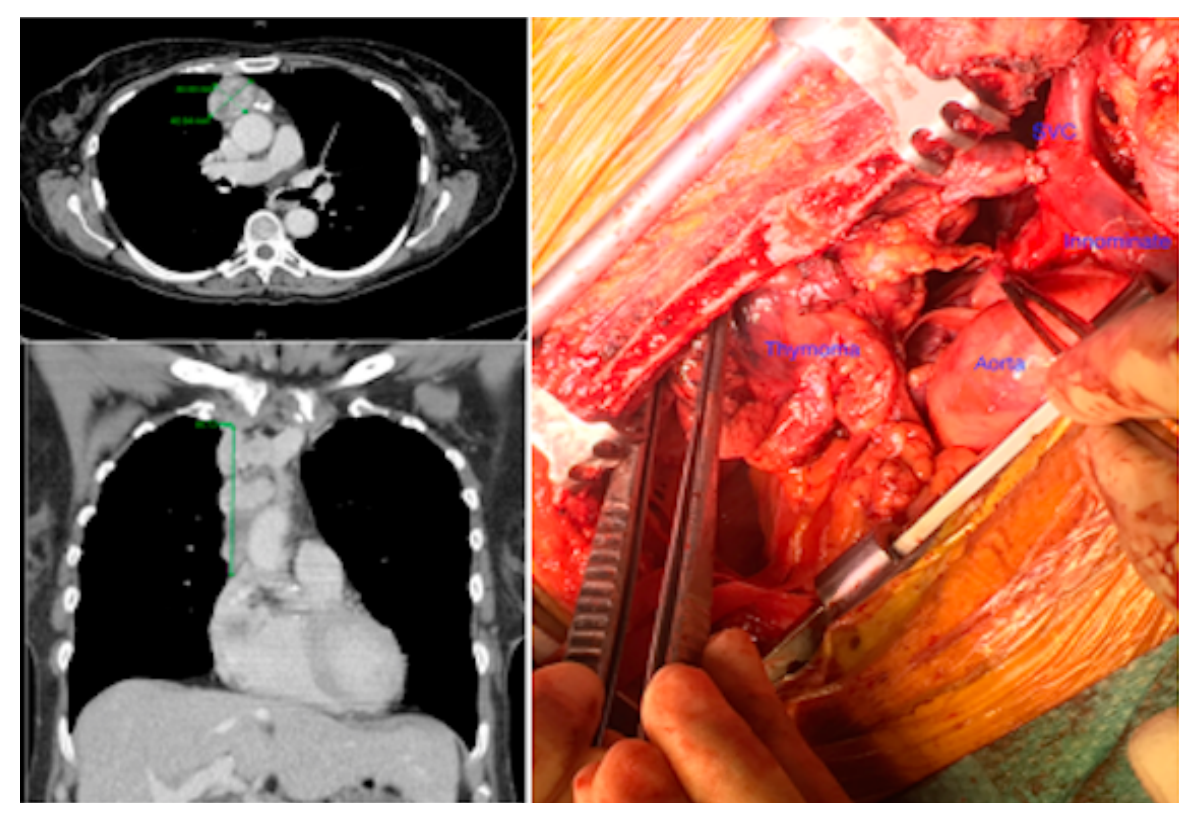

Figure 1: Pre-operative CT, Axial \& Coronal view showing $90 \mathrm{~mm}$ lesion invading the SVC confirmed at the intra operative picture.

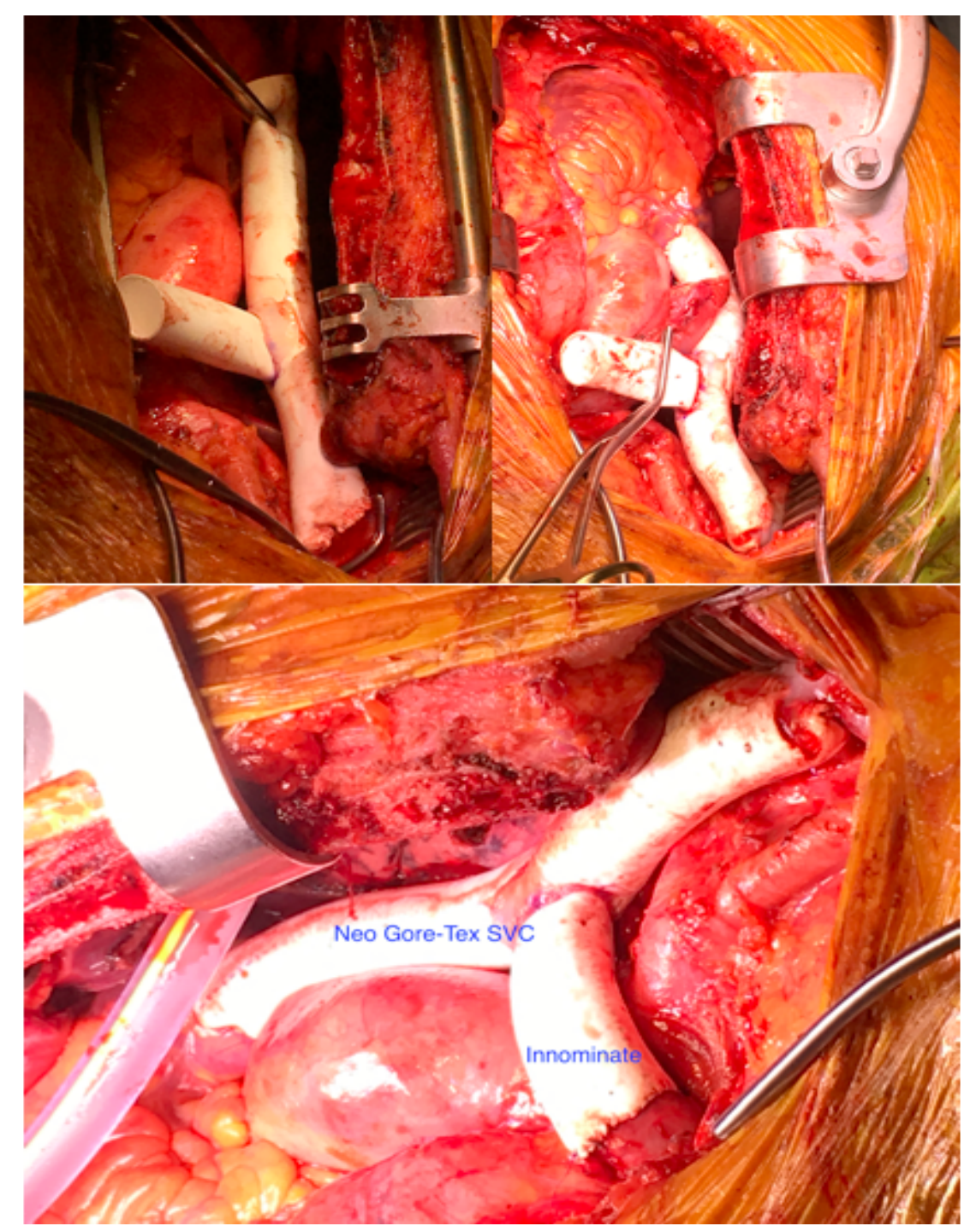

Figure 2: Serial SVC and Innominate reconstruction using Gore-Tex graft. 

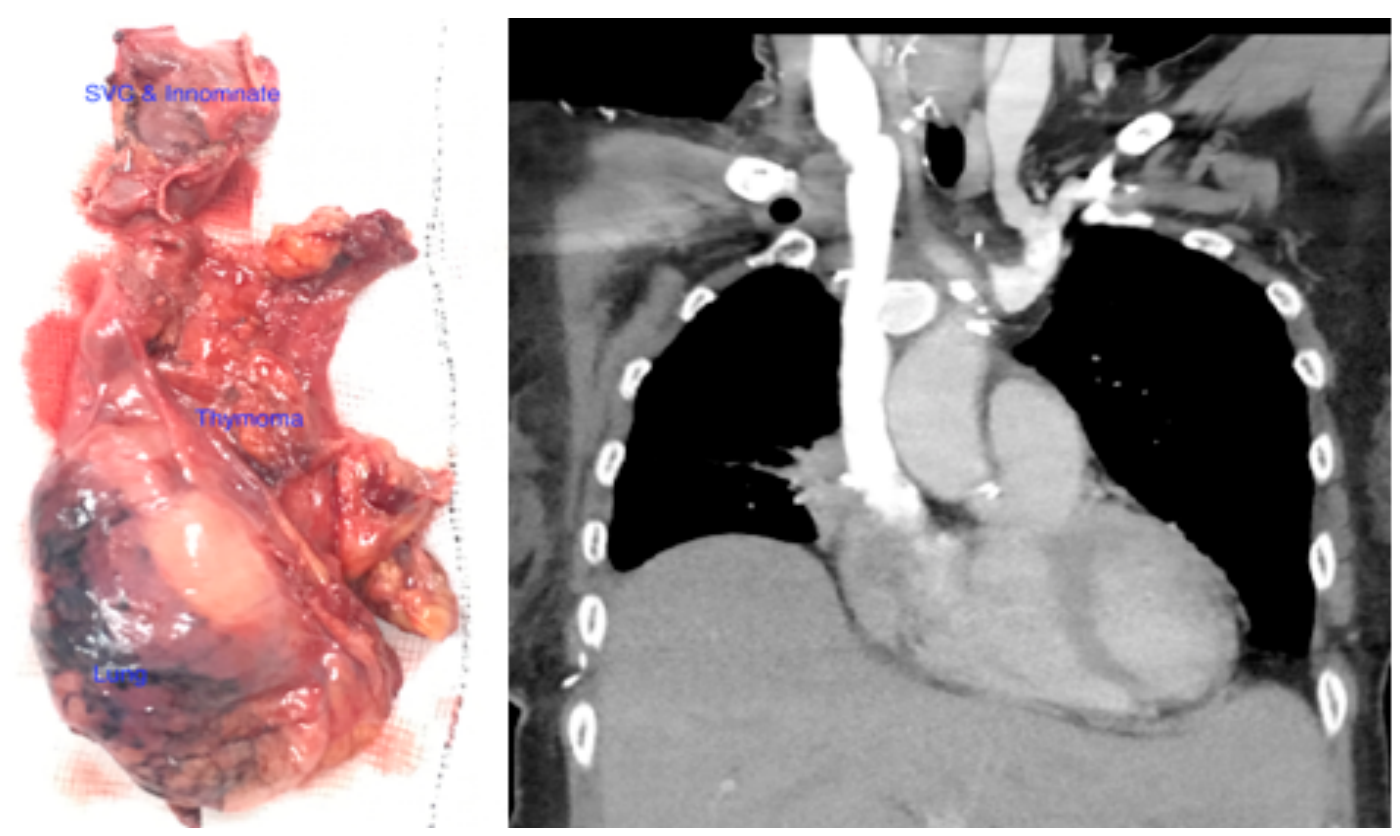

Figure 3: Enblock excision of thymoma, lung, phrenic, SVC, innominate and pericardium. Post-op CT scan with patent graft.

vading the great vessels is feasible with good outcome and should be considered in suitable patients.

\section{Comment}

MRI can provide excellent images to demonstrate mediastinal invasion [2]. The surgery is the mainstay of treatment and can be approached from a variety of ways [3]. The management for stage II and III is followed by adjuvant radiotherapy to decrease the local recurrence $[4,5]$. The timing of surgery is a controversial subject in cases of large invading thymomas. The induction chemotherapy may help down staging the disease in advanced cases [6]. The neoadjuvant therapy although may reduce the tumor mass but makes surgery more challenging as it changes the architecture and we would not advocate it from personal experience. The nodal dissection should be performed to define the staging, further management and prognosis. ITMIG recommends routinely marking and orientation of the specimen as well as sketch and photo of mounted specimen. Tumor staging and completeness of resection have been identified as significant factors but the presence of an associated systemic disease is a poor prognostic factor [1,7]. The outcome is promising with 20 -year survival for stage III being $49 \%$ and all thymoma patients should have lifelong follow up [8].

\section{Funding}

This project received no funding from any source.

\section{References}

1. Detterbeck, Frank C (2010) Synchronous, Separate, and Similar. Journal of Thoracic Oncology 6: S1689-S1690.

2. Sperling B, Marschall J, Kennedy R, et al. (2003) Thymoma: A review of the clinical and pathological findings in 65 cases. Can $J$ Surg 46: 37-42.

3. Brown, Lisa M (2017) Robot-assisted total thymectomy: How I teach it. The Ann Thorac Surg 103: 369-372.

4. Rimner A, Yao X, Huang J, et al. (2016) Postoperative radiation therapy is associated with longer overall survival in completely resected stage II and III thymoma-an analysis of the international thymic malignancies interest group (ITMIG) retrospective database. J Thorac Oncol 11: 1785-1792.

5. Ruffini E, Detterbeck F, VanRaemdonck D, et al. (2014) Tumours of the thymus: A cohort study of prognostic factors from the European Society of Thoracic Surgeons database. Eur J Cardiothorac Surg 46: 361-368.

6. Kunitoh H, Tamura T, Shibata T, et al. (2010) A phase II trial of dose-dense chemotherapy, followed by surgical resection and/ or thoracic radiotherapy, in locally advanced thymoma: Report of a Japan. Br J Cancer 103: 6-11.

7. Yuan Z-Y, Gao S-G, Mu J-W, et al. (2017) Long-term outcomes of 307 patients after complete thymoma resection. Chin J Cancer 36: 46.

8. Moser B, Fadel E, Fabre D, et al. (2017) Surgical therapy of thymic tumours with pleural involvement: An ESTS thymic Working Group Project. Eur J Cardiothorac Surg 52: 346-355.

DOI: $10.36959 / 582 / 424$ 